\title{
Serum Pharmacochemistry Analysis Using UPLC-Q-TOF/MS after Oral Administration to Rats of Shenfu Decoction
}

\author{
Jia-le He, ${ }^{1,2}$ Jia-wei Zhao, ${ }^{1,2}$ Zeng-chun $\mathrm{Ma}^{2}$ Yu-guang Wang, ${ }^{2}$ Qian-de Liang, \\ Hong-ling Tan, ${ }^{2}$ Cheng-rong Xiao, ${ }^{2}$ Xiang-lin Tang, ${ }^{2}$ and Yue Gao ${ }^{2}$ \\ ${ }^{1}$ Graduate School, Anhui Medical University, Hefei, China \\ ${ }^{2}$ Beijing Institute of Radiation Medicine, Tai-Ping Road 27, Beijing 100850, China
}

Correspondence should be addressed to Zeng-chun Ma; mazchun@139.com and Yue Gao; gaoyue@bmi.ac.cn

Received 23 October 2014; Revised 27 May 2015; Accepted 28 May 2015

Academic Editor: Kazuo Toda

Copyright (C) 2015 Jia-le He et al. This is an open access article distributed under the Creative Commons Attribution License, which permits unrestricted use, distribution, and reproduction in any medium, provided the original work is properly cited.

\begin{abstract}
The purpose of this study was to study the serum pharmacochemistry of SFD as well as the material basis through analyzing the constituents absorbed in blood. The SFD was orally administrated to Wistar rats at $20 \mathrm{~g} \cdot \mathrm{kg}^{-1}$, and Ultra Performance Liquid Chromatography (UPLC) fingerprints of SFD were created. Serum samples were collected for analysis, and further data processing used MarkerLynx XS software. 19 ginsenosides and 16 alkaloids were detected in SFD. The absorption of alkaloids (mainly monoester diterpenoid alkaloids) increased when Aconitum carmichaeli Debx. was combined with Panax ginseng, while the ginsenosides remained stable. Diester diterpenoid alkaloids were not present in the serum samples. A suitable serum pharmacochemistry method was successfully established to study pharmacological effects and potential improvements in formulation. This may also be useful for toxicity reduction. We suspect that the increased absorption of the monoester diterpenoid alkaloids from the mixture of Panax and Radix, compared to the Panax only extract, may be the reason for the combination of the two herbs in popular medicine formulas in China.
\end{abstract}

\section{Introduction}

Shenfu Decoction (SFD) consisting of an equal ratio of ginseng root (radix ginseng, Renshen) and aconite root (Radix Aconiti Lateralis Preparata, Fuzi) is an example of a classic Chinese traditional herb-couple formulation, where two herbs are prescribed together to decrease toxicity and/or increase efficacy. For example, inclusion of Glycyrrhiza uralensis can prevent or destroy the toxicity of treated Fuzi [1]. The main components are ginsenosides and alkaloids. SFD was originally described in Ji Sheng Xu Fang (1253 in western calendar) written by Yonghe in the Song Dynasty. In many formulae of Traditional Chinese Medicine (TCM), Renshen is frequently prescribed in combination with other herbs to decrease toxicity and increase efficacy. Ginsenosides are the main bioactive constituents in the famous Chinese herb Renshen. They include protopanaxadiol, protopanaxatriol, octotillol, and oleanolic acid. Aconitines are the main constituents in Aconitum carmichaelii Debx. Aconitines include monoester diterpenoid alkaloids (MDAs) and diester diterpenoid alkaloids (DDAs) and have differences in esterification. Their chemical structures are shown in Figure 1. Due to the high toxicity of DDAs, Fuzi is combined with Renshen to decrease its toxicity.

SFD is used to treat cardiovascular diseases such as circulatory collapse, shock, thoracic obstruction, and acute thoracic pain. It has a therapeutic effect on heart failure and ischemia-reperfusion injury [2]. One study showed that blockage of the sodium channels in cardiac myocytes may be one of the important molecular mechanisms of its cardiac effect [3]. There are no detailed studies of its mechanism of action, and the bioactive compounds that account for its therapeutic effects remain unclear.

While they have the so-called active ingredients, there are no empirical data to prove its effectiveness. Oral administration is one of the primary modes for TCM. The bioactive compounds are absorbed in the blood and transferred to the 
<smiles>[R20]C1CCC2(C)C(CCC3(C)C2CC(O)C2C(C([Y])(I)CCC=C(C)C)CCC23I)C1(C)C</smiles>

20(S)<smiles>[R20]C1CCC2(C)C(CCC3(C)C2CC(O)C2C(C([R20])(C)CCC=C(C)C)CCC23I)C1(C)C</smiles>

$20(\mathrm{R})$

(a)<smiles>[R17]OC1CC2(C)C(CC(O[R20])C3C(C(I)(CCC=C(C)C)CCC=C(C)C)CCC32I)C2(C)CCC(O)C(C)(C)C12</smiles>

$20($ S)<smiles>[R10]C(C)(CCC=C(C)C)C1CCC2(I)C1C(O)CC1C(C)(C)C(O)CCC12C</smiles>

$20(\mathrm{R})$

(b)<smiles>CC1(C)CCC2(C(=O)OC3OC(CO)C(O)C(O)C3O)CCC3(I)C(=CCC4C5(C)CC(OC6OC(C(=O)O)C(O)C(O)C6O)CC(C)(C)C5CCC43C)C2C1</smiles>

(c)

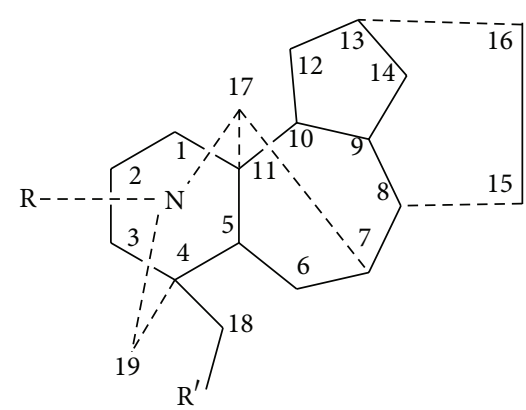

(d)

FIGURE 1: The chemical structure of 20(S)- and 20(R)-protopanaxadiol ginsenosides (a), 20(S)- and 20(R)-protopanaxatriol ginsenosides (b), oleanolic acid saponins type (c), and aconitines (d).

target. Thus, while there are multiple components in herbs, only those that are absorbed into circulation are effective [4].

Pharmacokinetics remains unresolved in the use of Chinese herbs. In our previous research, we focused on the influence of DDAs, drug metabolism enzymes, and transporters after the combination of Renshen and Fuzi [5-7]. Our previous study in vitro showed that there were significant differences between codecoction and mixed decoction of Renshen and Fuzi [5]. The content of toxic alkaloids was higher in mixed decoction than codecoction which was probably the attenuation basis. To confirm whether they behaved similarly in vivo is one of our goals. Here, we developed a rapid and sensitive Ultra Performance Liquid Chromatography/Quadrupole Time-of-Flight Mass Spectrometry 
(UPLC/Q-TOF-MS) method to study the pharmacokinetics of SFD constituents.

\section{Materials and Methods}

2.1. Chemicals and Materials. HPLC-grade acetonitrile was purchased from Fisher Scientific (Waltham, MA, USA). Methanol was purchased from Sinopharm Chemical Reagent Co. (Shanghai, China). Formic acid was HPLC-grade (CNW Technologies GmbH, Dusseldorf, Germany). Renshen and Fuzi were purchased from Hebei Anguo Drug Market (Hebei, China) and authenticated by Professor Baipin Ma, Institute of Radiation Medicine Sciences of the Academy of Military Medical Sciences. The Renshen was at least 5-year growth white ginseng which was processed by the method of the Chinese Pharmacopoeia (2010) and the Fuzi was nonprocessed Radix Aconiti Lateralis Preparata (only for laboratory use). The vouched specimens were stored in storage room of the Department of Pharmacology and Toxicology of the Institute of Radiation Medicine Sciences of the Academy of Military Medical Sciences.

2.2. Preparation of Decoction and for LC-MS Analysis. Renshen $(100 \mathrm{~g})$ was soaked in $800 \mathrm{~mL}$ distilled water for $30 \mathrm{~min}$ and decocted for $1 \mathrm{~h}$ and then filtered. The residue was decocted in $500 \mathrm{~mL}$ distilled water for $1 \mathrm{~h}$ and filtered. The filtrate was then combined and labeled Renshen decoction. Fuzi $(100 \mathrm{~g})$ was decocted in $800 \mathrm{~mL}$ distilled water for $10 \mathrm{~min}$ and then filtered. The residue was decocted in $500 \mathrm{~mL}$ distilled water for $30 \mathrm{~min}$ and filtered and labeled Fuzi decoction. Then two different kinds of decoctions were prepared, namely, Renshen-Fuzi codecoction where the two herbs mixed together were extracted with water and Renshen-Fuzi mixed decoction where the individual herbs were extracted separately with water and the extracts then mixed together. The decoctions were evaporated to $1 \mathrm{~g}$ crude herb per $\mathrm{mL}$. The decoctions were stored at $4^{\circ} \mathrm{C}$, and the decoction samples were subjected to UPLC/Q-TOF-MS analysis, and the datasets were processed with MassLynx software.

2.3. Animals Handling and Serum Samples Preparation. Forty male Wistar rats $(200 \pm 20 \mathrm{~g})$ were obtained from the Laboratory Animal Center of the Academy of Military Medical Sciences (Beijing, China, production certificate number SCXK-(M) 2007-004). All procedures were performed in accordance with the protocol outlined in the Guide for the Care and Use of Laboratory Animals published by the US National Institute of Health (NIH publication number 8523, revised 1996) and approved by the Committee on the Ethics of Animal Experiments of the Academy of Military Medical Sciences. They were randomly divided into 5 groups: blank group (B-Group), Renshen decoction group (R-Group), Fuzi decoction group (F-Group), codecoction group (CGroup), and mixed decoction group (M-Group). There were 8 rats in each group. The rats were kept in an animal room with controlled environment (temperature: $22 \pm 2^{\circ} \mathrm{C}$, relative humidity: $55 \pm 5 \%$, and $12 \mathrm{~h}$ light-dark cycle) for 3 days before the experiment. All animals were free to access distilled water and standard food.

Every group except the blank group received an oral administration of $20 \mathrm{~g} \cdot \mathrm{kg}^{-1}$ decoction for 3 days. A distilled water vehicle control was given to the blank group. Blood samples $(500 \mu \mathrm{L})$ were collected from the retroorbital sinus $1 \mathrm{~h}$ after oral administration on the 3rd day and centrifuged at $3000 \mathrm{rpm}$ for $10 \mathrm{~min}$. Then, $800 \mu \mathrm{L}$ methanol was added to the $200 \mu \mathrm{L}$ serum samples, vortexed, and then centrifuged at $13000 \mathrm{rpm}$ for $10 \mathrm{~min}$. The supernatant solution was transferred to another tube and dried with nitrogen gas. The residue was stored in $50 \%$ acetonitrile $(200 \mu \mathrm{L})$ and frozen at $-80^{\circ} \mathrm{C}$ until analysis.

\subsection{Instrumentation and Chromatographic Conditions}

2.4.1. Ultra Performance Liquid Chromatography. Chromatographic analysis was performed with an ACQUITY Ultra Performance Liquid Chromatography system (Waters, USA) controlled with MassLynx V4.1 software. Separation used an ACQUITY UPLC HSS T3 Column $(2.1 \times 100 \mathrm{~mm}, 1.8 \mu \mathrm{m}$, Waters). Water with $0.1 \%$ formic acid $(\mathrm{v} / \mathrm{v})$ and acetonitrile with $0.1 \%$ formic acid $(\mathrm{v} / \mathrm{v})$ were used as mobile phases $\mathrm{A}$ and $\mathrm{B}$, respectively, at a flow rate of $0.45 \mathrm{~mL} \cdot \mathrm{min}^{-1}$. The gradient conditions of the mobile phase in positive mode were 0 2 min: 5\% B; $2-6$ min: 5-12\% B; 6-8 min: $12-20 \%$ B; 8-16 min: $20-50 \%$ B; $16-17$ min: $50-5 \%$ B; and $17-18 \min : 5 \%$ B. The gradient conditions of the mobile phase in negative mode were $0-1 \mathrm{~min}$ : $25 \% \mathrm{~B} ; 1-3 \mathrm{~min}$ : $25-30 \% \mathrm{~B} ; 3-16 \mathrm{~min}$ : $30-35 \%$ B; $16-17 \mathrm{~min}$ : $35-25 \% \mathrm{~B}$; and 17-18 min: $25 \% \mathrm{~B}$.

2.4.2. Mass Spectrometry. A Waters Synapt High-Definition Time-of-Flight Mass Spectrometry (TOF-MS) system (Waters) equipped with an electrospray ionization (ESI) source operating in positive and negative mode was connected to the UPLC. A capillary voltage of 2.9 and $3.0 \mathrm{kV}$ was used in positive and negative ionization mode, respectively. The desolvation temperature was $450^{\circ} \mathrm{C}$, and the sampling cone voltage was $40 \mathrm{~V}$. The extraction cone voltage was $4.0 \mathrm{~V}$, source temperature was $100^{\circ} \mathrm{C}$, and cone gas flow was $50 \mathrm{~L} \cdot \mathrm{h}^{-1}$. The desolvation gas flow rate was $900 \mathrm{~L} \cdot \mathrm{h}^{-1}$ in both positive and negative ionization modes. The mass was corrected during acquisition with leucineenkephalin to generate a reference ion at $m / z 556.2771 \mathrm{Da}$ $\left([\mathrm{M}+\mathrm{H}]^{+}\right)$in positive ion mode and $m / z 554.2615 \mathrm{Da}([\mathrm{M}-$ $\mathrm{H}]^{-}$) in the negative ion mode. This ensured accurate mass measurements.

2.5. Data Analysis. All data were processed by MassLynx V4.1 software (Waters). The chromatographic peaks were integrated, aligned, and combined with accurate mass to charge ratios. A reference retention time was found for each expected compound. Using our previous work, the compounds in the decoction were identified [8-10]. The data were further processed by MarkerLynx XS software (Waters). The exported data list, partial least-squares-discriminant analysis (PLSDA) as well as principal component analysis (PCA), was used to analyze the differences between the groups. 


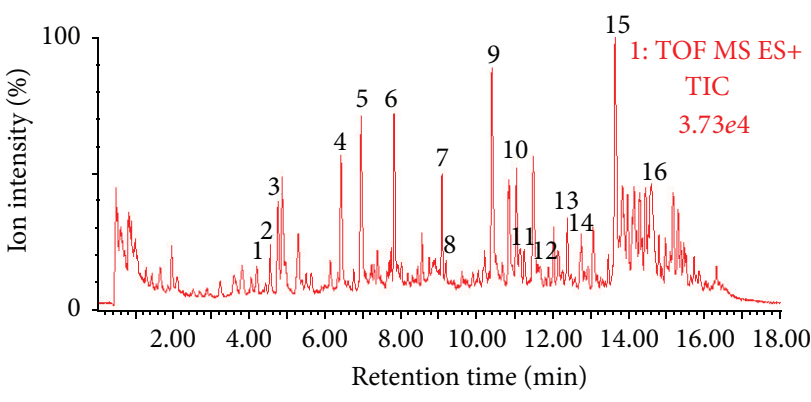

(a)

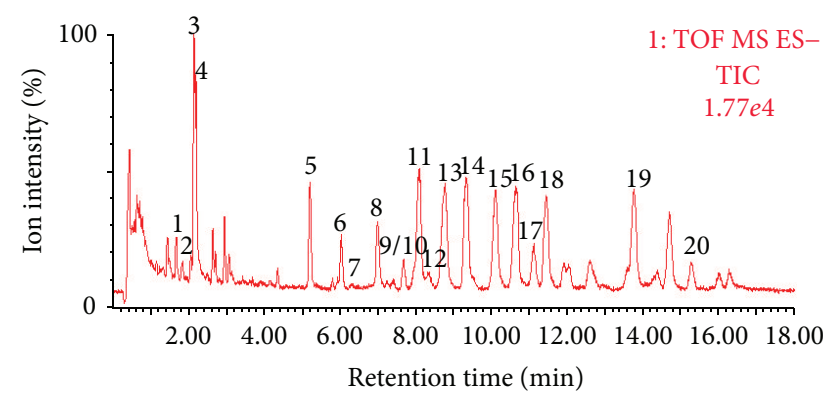

(b)

FIgURE 2: Total ion chromatogram of codecoction. (a) Positive ion mode detected alkaloids, (b) negative ion mode detected ginsenosides.

\section{Results}

3.1. Chemical Analysis of Codecoction. The ginsenosides and alkaloids in SFD were identified using UPLC combined with a TOF-MS detector. The total ion chromatogram of SFD in positive and negative ion modes is shown in Figure 2 and was processed with MassLynx V4.1 (Waters, USA). Comparing the retention time and mass data with reference compounds identified the target compounds. Both the MDAs and DDAs of alkaloids and 20(s)-protopanaxadiol type, 20(s)-protopanaxatriol type, and oleanolic acid saponin-type ginsenosides were detected in SFD. The chemical compositions are shown in Tables 1 and 2.

\subsection{Alkaloids Difference in Serum Detected by Positive Mode}

3.2.1. Differences in the Five Groups. All five groups were processed by PLS-DA and were used to highlight variation among the five groups (see Figure 3). Exported PCA plots and loading plots showed that the five groups formed five clusters. This indicated that the components in the serum were different.

3.2.2. Alkaloids Difference between B-Group, F-Group, MGroup, and C-Group. Comparing F-Group with B-Group, 14 kinds of alkaloids including DDAs, MDAs, and general alkaloids in aconitum plants were detected. Most of them were trace amount, which indicated the alkaloids poor absorption in vivo. The main reason was high efflux ratio reduced by P-glycoprotein [11-13]. These 14 kinds of alkaloids might be the chemical constituents involved in therapeutic efficacy of SFD. Some researchers thought that Fuzi's therapeutic activity (anti-inflammatory, analgesic, and cardiotonic activity) seems to have relevance to the presence of toxic alkaloids $[14,15]$ but the other researchers thought that Fuzi's toxicity compounds (mainly aconitine, mesaconitine, and hypaconitine) are not essential for its efficacy [16] (see Figure 4).

After detecting these 14 alkaloids, we further processed the data. Comparing C-Group with F-Group, the absorption of MDAs (benzoylaconine, mesaconine, 10-OHbenzoylmesaconine, and dehydrated benzoylmesaconine) decreased and the general alkaloids in aconitum plants (cammaconine, neoline, and talatizamine) increased. Most alkaloids (cammaconine, carmichaeline, talatizamine, acetyltalatizamine, and dehydrated benzoylhypaconine) absorption in M-Group was increased rather than F-Group. The increased or decreased absorption of alkaloids may contribute to the attenuation and synergistic effects. The decreased absorption of toxic alkaloids leads to attenuation and increased nontoxic alkaloids contribute to synergistic effects mainly because of compatibility of Renshen. The phenomenon was found in ancient year by the Chinese and we make it clear by analyzing the chemical compounds (see Figures 5 and 6).

The M-Group data showed that all alkaloids were absorbed more than the C-Group. The relative intensity of the ion indicated that the toxic chemicals (mesaconine, 10-OH-benzoylmesaconine, dehydrated benzoylmesaconine, and dehydrated benzoylhypaconine) were less absorbed than most general alkaloids in Aconitum plants (cammaconine, carmichaeline, fuziline, talatizamine, acetyltalatizamine, and neoline). The content of toxic alkaloids was higher in mixed decoction than codecoction which was probably the attenuation basis in vitro, and the results in vivo showed a similar consequence which indicated that the Renshen attenuated the toxicity of Fuzi. And ester exchange or degradation reactions occurring during the processing of codecoction with Renshen were considered to be the key factor of attenuation [5] (see Figure 7).

3.2.3. Ginsenosides Differences in the Serum in Negative Mode. The ginsenosides and alkaloids were detected in negative and positive mode, respectively. In our study, the codecoction and mixed decoction had small differences in the negative mode. This indicated that the ginsenosides behaved similarly, and the results presented the same tendency with an unpublished paper for the negative result in vitro. The same processing method used in positive mode was also applied to negative mode to analyze the ginsenoside differences. The PCA and PLS-DA/S-Plot showed no obvious distinction between the C-Group and the M-Group. However, 13 kinds of ginsenosides $\left(\mathrm{GRg}_{1}, \mathrm{GRe}, \mathrm{GRd}, \mathrm{GRo}, \mathrm{GRc}, \mathrm{GRb}_{2}, \mathrm{GRb}_{3}, \mathrm{GRb}_{1}\right.$, $\mathrm{GRa}_{2}, \mathrm{GRa}_{1}$, MalonylGRc, MalonylGRb , and MalonylGRb $_{2}$ ) were detected in the serum of the Panax ginseng group. The extracted ion chromatogram and mass spectrogram of the ginsenosides are shown in Figures 8, 9, and 10. 


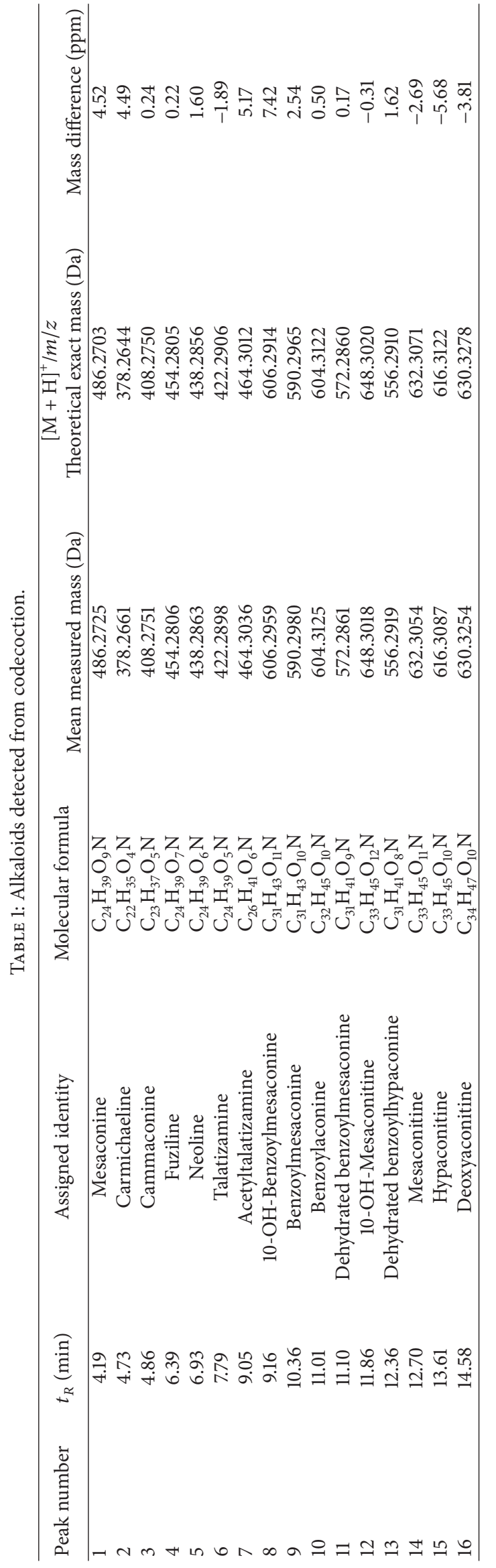




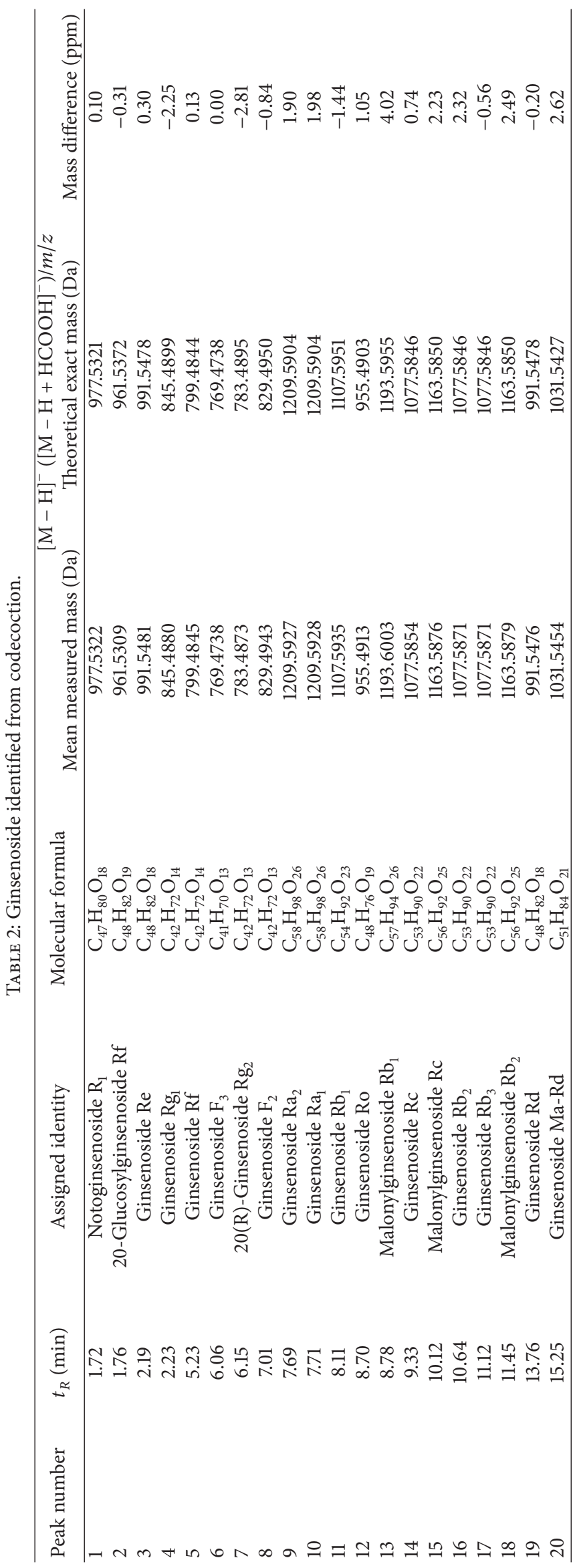




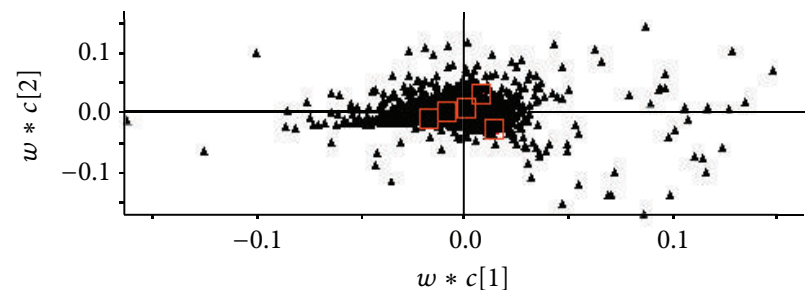

- X variables

$\star$ Responses

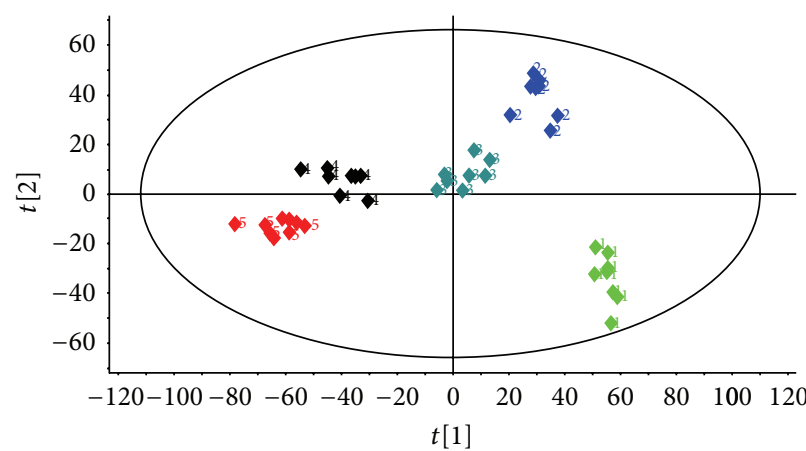

$$
\begin{array}{ll}
\text { 1 B-Group } & \bullet \text { R-Group }
\end{array} \text { - } 4 \text {-Group }
$$

EZinfo 2-MyStudy467 (M3: PLS-DA)-2014-03-11 03:19:04 (UTC + 8)

(a)

(b)

FIGURE 3: (a) PLS-DA loadings plot of five groups. (b) PCA score plot of the five groups. B-Group: blank group; R-Group: Renshen decoction group; F-Group: Fuzi decoction group; C-Group: codecoction group; M-Group: mixed decoction group. There were 8 rats in each group and each received an oral administration of $20 \mathrm{~g} \cdot \mathrm{kg}^{-1}$ decoction for 3 days. The blank group received an equivalent volume of distilled water.

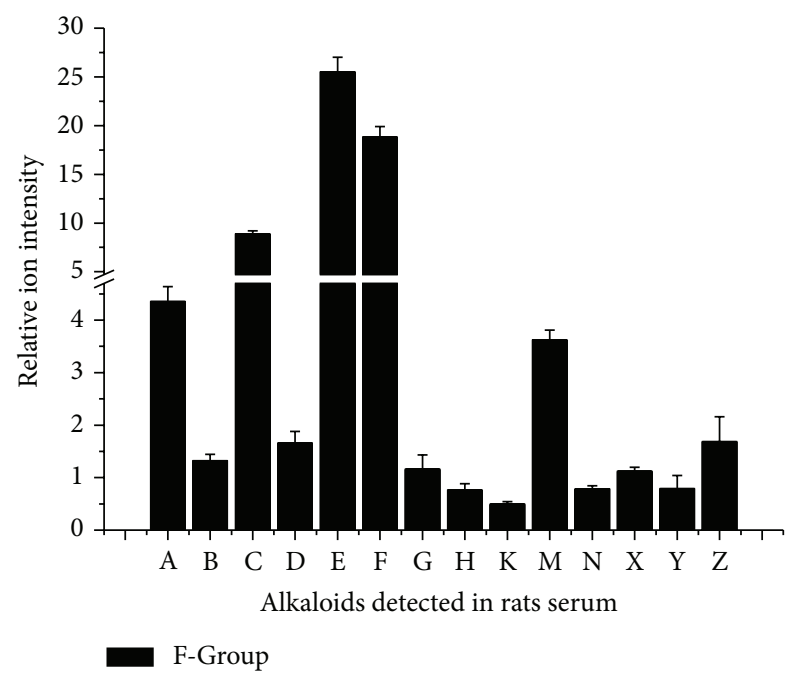

FIGURE 4: Relative content of alkaloids detected in F-Group serum. F-Group: Fuzi decoction group; A: cammaconine; B: carmichaeline; C: fuziline; D: neoline; E: talatizamine; F: acetyltalatizamine; G: mesaconine; $\mathrm{H}$ : 10-OH-benzoylmesaconine; $\mathrm{K}$ : benzoylaconine; $\mathrm{M}$ : benzoylmesaconine; $\mathrm{N}$ : dehydrated benzoylmesaconine; X: dehydrated benzoylhypaconine; Y: 10-OH-mesaconitine; Z: hypaconitine.

\section{Discussion}

Researchers have reported quantitative analysis of aconitum alkaloids of SFD in vitro as well as the pharmacokinetic behavior of Fuzi and drug-drug interaction mechanisms in herb pair decoctions [17-19]. Relatively few studies have been conducted on the material basis of SFD in vivo, and most researchers focused on Shenfu injection which is a kind of processed Shenfu formulation $[20,21]$. The material basis of toxicity reduction and pharmacological effect improvement

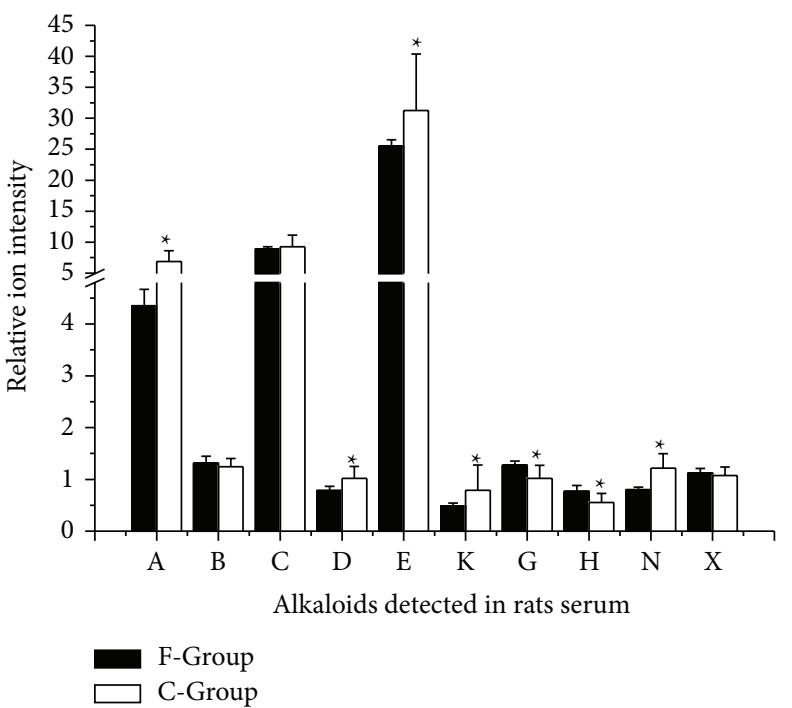

FIGURE 5: Relative content of alkaloids detected in rats serum of F-Group and C-Group. F-Group: Fuzi decoction group; C-Group: codecoction group. A: cammaconine; B: carmichaeline; C: fuziline; D: neoline; E: talatizamine; K: benzoylaconine; G: mesaconine; $\mathrm{H}$ : 10-OH-benzoylmesaconine; $\mathrm{N}$ : dehydrated benzoylmesaconine; X: dehydrated benzoylhypaconine.

in vivo remains unclear. In previous studies, the attenuation and synergistic effects of SFD including decoction time and herb ratio were studied in vitro. However, aconitum alkaloids serum levels were difficult to detect after oral administration, and only trace level was seen for its low bioavailability [18, 22]. Only Shenfu injected powder showed detectable alkaloids in serum [23]. Multicomponents and multitarget herbs resulted in complex systems with difficult detection. Recently, UPLC coupled with TOF-MS has become a vitally important tool in studying Chinese herbs. It is fast with good selectivity, high 


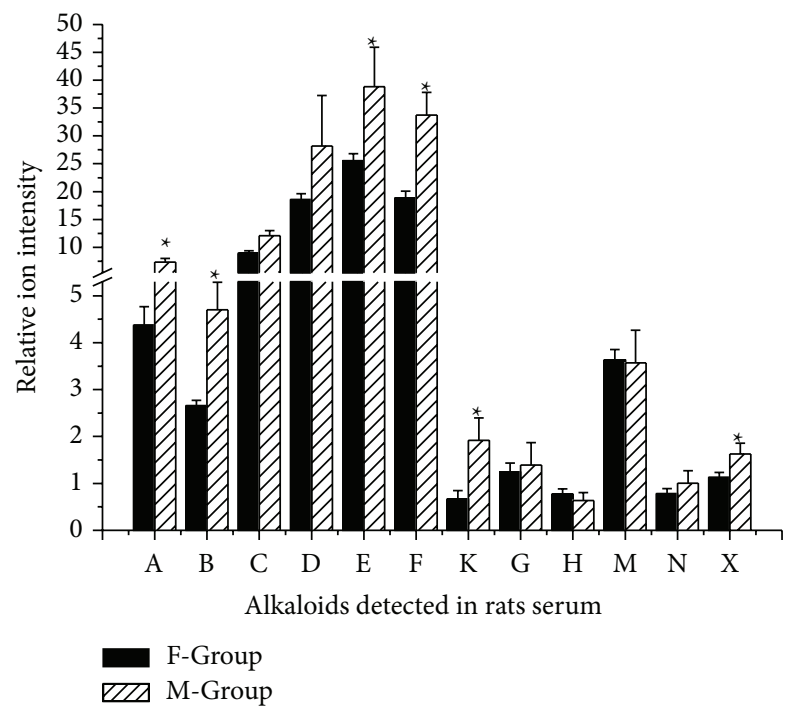

FiguRE 6: Relative content of alkaloids detected in rats serum of F-Group and M-Group. F-Group: Fuzi decoction group; M-Group: mixed decoction group. A: cammaconine; B: carmichaeline; C: fuziline; D: neoline; E: talatizamine; F: acetyltalatizamine; K: benzoylaconine; G: mesaconine; H: 10-OH-benzoylmesaconine; M: benzoylmesaconine; $\mathrm{N}$ : dehydrated benzoylmesaconine; X: dehydrated benzoylhypaconine.

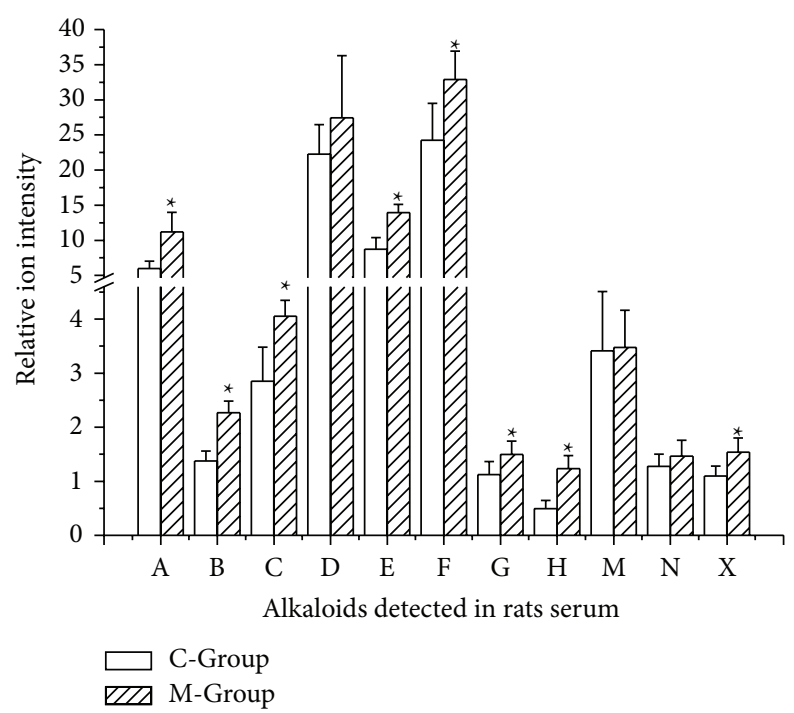

Figure 7: Relative content of alkaloids detected in rats serum of C-Group and M-Group. C-Group: codecoction group; M-Group: mixed decoction group. A: cammaconine; B: carmichaeline; C: fuziline; D: neoline; E: talatizamine; F: acetyltalatizamine; G: mesaconine; H: 10OH-benzoylmesaconine; M: benzoylmesaconine; N: dehydrated benzoylmesaconine; X: dehydrated benzoylhypaconine.

resolution, and accurate mass measurements. This makes it attractive for the analysis of complex biological sample [24].

Shenfu formulation was a classical Chinese medicine for its obvious therapy efficiency on heart fail [25], but Fuzi in the formulation was a famous toxicity herb for its severe arrhythmia and neurological, cardiovascular, and gastrointestinal symptoms [26]. The herb pair was used for synergism and attenuation and the decoction method was thought to be the main reason for its chemical difference, but whether it behaves similarly in vivo was still a mystery, and here we approved an evidence to evaluate the Shenfu formulation in vivo. The concentration of hypaconitine and deoxyaconitine decreased, while benzoylmesaconine, benzoylhypaconine, and dehydrated benzoylmesaconine increased in the codecoction samples. The toxicity increased in single decocted or mixed decocted samples [5]. The codecoction inhibited toxic compounds, and the inhibited alkaloids dissolution resulted in a lower alkaloids content in the C-Group than the M-Group. They behaved similarly in vivo and in vitro. Here, Renshen showed evidence of increasing the absorption of alkaloids. These alkaloids were measured in a relative concentration range and found to be in accordance with the 


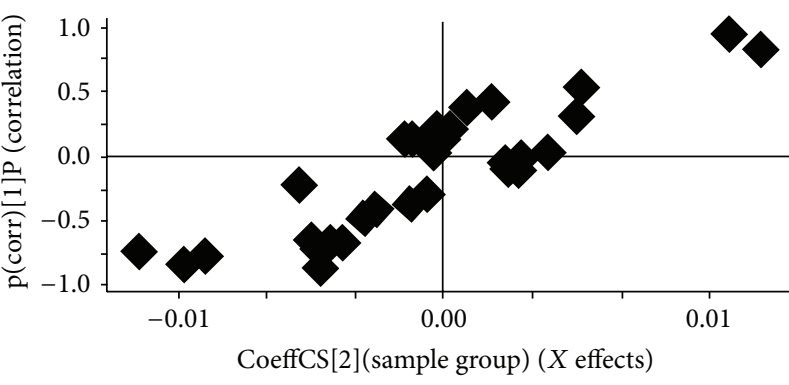

(a)

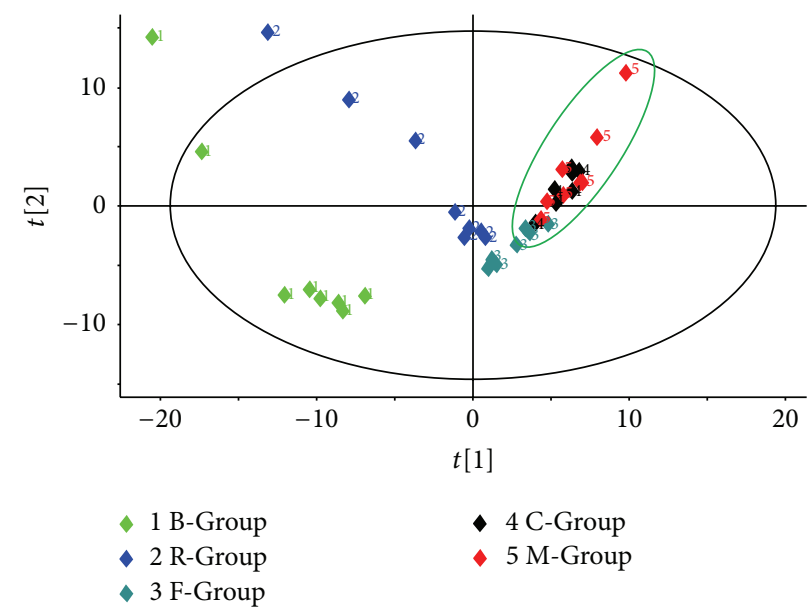

(b)

Figure 8: (a) PCA scores plot of C-Group and M-Group. (b) PLS-DA loadings plot of five groups. B-Group: blank group; R-Group: Renshen decoction group; F-Group: Fuzi decoction group; C-Group: codecoction group; M-Group: mixed decoction group. There were 8 rats in each group and each received an oral administration of $20 \mathrm{~g} \cdot \mathrm{kg}^{-1}$ decoction for 3 days. The blank group received an equivalent volume of distilled water.

tradition of TCM. We suspect that toxicity reduction is due to decoction in vitro; the pharmacological effect improvement is due to increased absorption and metabolism in vivo.

The pharmacological effect in vivo was affected by absorption and metabolism. In previous study, alkaloids were metabolized by Cytochrome P450 (CYP450) 3A [27]. A study on Shenfu injection indicated that it inhibited the enzyme activity of CYP3A at the mRNA level [6]. Mean Residence Time (MRT) increased when the enzyme activity was inhibited. Its absorption increase contributed to an increase in the Area under the Curve (AUC). The increased MRT and AUC indicated an increase in pharmacological effects.

Efflux transporters in the intestine play an important role in absorption. They also protect the body from toxin damage. The ATP-binding cassette transporters include Pglycoprotein (P-gp), multidrug resistance-associated protein isoform 2 (MRP2), and breast cancer resistance protein (BCRP). They modulate the absorption, distribution, metabolism, and excretion of medicine. These proteins are highly expressed in the apical membranes of intestinal epithelial, hepatic, and renal tubular cells. Transport studies show that the efflux ratios of aconitine, mesaconitine, and hypaconitine were significantly elevated due to P-gp and BCRP [11]. Panax ginseng and ginsenosides like ginsenoside $\mathrm{F} 1$, ginsenoside $\mathrm{Re}$, and ginsenoside $\mathrm{Rb}_{2}$ induced the function of P-gp [28]. An important clue is seen in that Fuzi's toxicity compounds (aconitine, mesaconitine, and hypaconitine) are not essential for its efficacy [16]. While aconitine, mesaconitine, and hypaconitine were not detected in serum, it could be because they were below the detection limit.

The difference in molecular structure between DDAs and MDAs is an acetyl group. This caused the different efflux ratios. The lost acetyl group decreased the toxicity of the DDAs and the efflux ratio [29]. In another TCM herb pair Fuzi-Ganjiang formula, Ganjiang (Rhizoma Zingiberis, derived from the dry rhizome of Zingiber officinale Rosc.) was combined with Fuzi to decrease its toxicity and improve pharmacological effects. The authors showed that Ganjiang enhanced the absorption of MDAs and promoted the elimination of DDAs [30]. We could hypothesize that Renshen played the same role in these herb pairs. Our findings showed that nontoxic alkaloids in the M-Group and C-Group absorption increased more than F-Group. The absorption of alkaloids in mixtures or extractions was better than that of pure compounds seen in other experiments [12].

Like many other TCMs, SFD is always taken orally. Ginsenosides showed poor absorption and low bioavailability [31]. Major factors that limited the intestinal absorption of ginsenosides were poor membrane permeability and active biliary excretion. This limited systemic exposure to most ginsenosides and their deglycosylated metabolites [32]. The poor absorption and bioavailability of ginsenoside make them difficult to accurately measure in serum. Thus, we successfully developed a new method to measure ginsenoside previously. The different clearance rate of ginsenosides is considered to be the mechanism of Shenfu injection [10].

In the past decades, researchers have studied traditional Chinese formulas sufficiently, but the material basis and mechanism are still unclear with most data focusing on pharmacokinetics. Most studies are multicomponent herbs and with multiple targets in vivo. In addition to active compounds, there are also inactive and even toxic compounds in herbs. The material basis, pharmacology, component compatibility, and physiological disposition of TCM remain unclear. Understanding the attenuation and synergistic effects on absorption is an important goal of this study. Here, we studied the material basis of attenuation and synergistic effects by measuring the absorbed ingredients. 

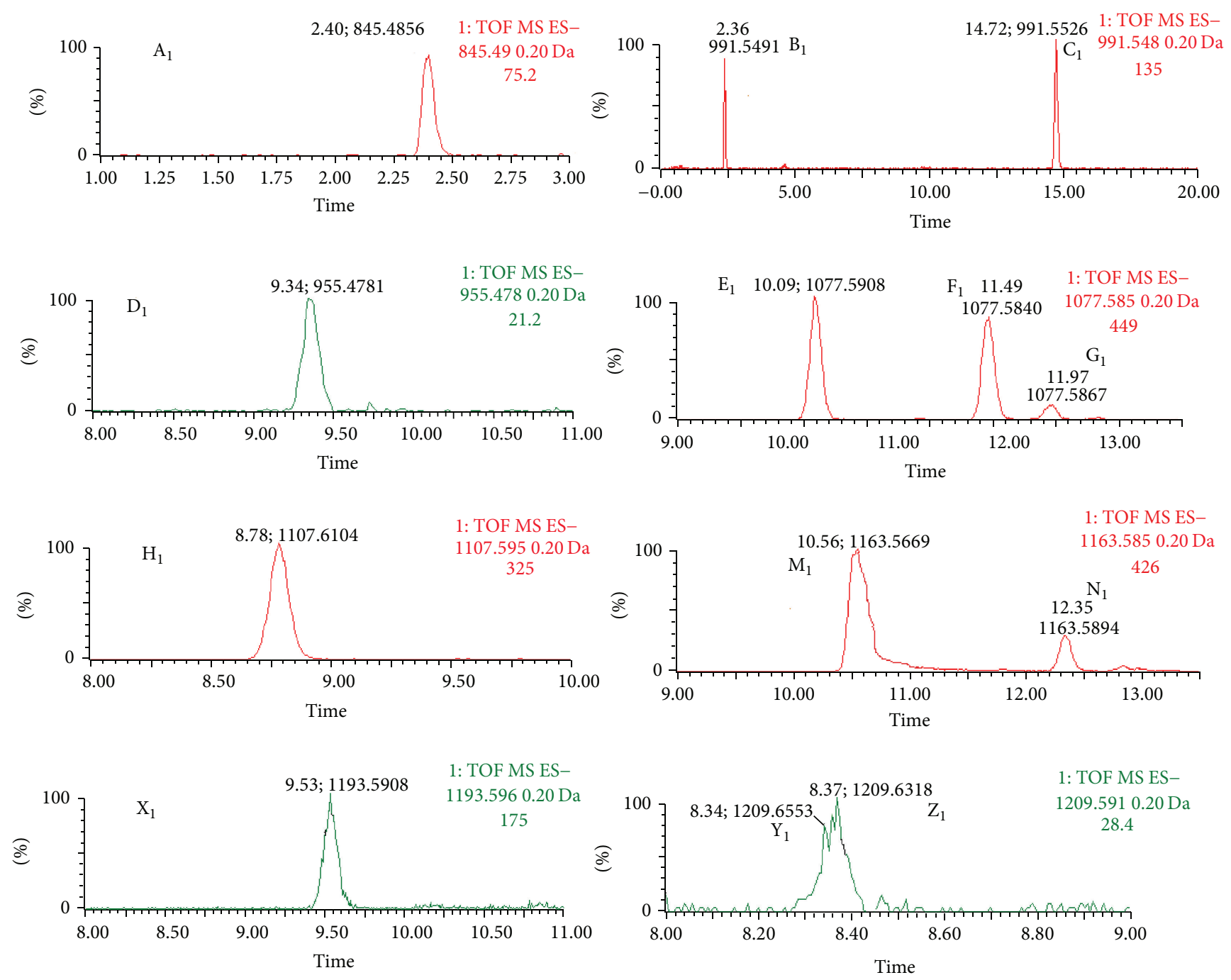

FIGURE 9: Extracted ion chromatogram of ginsenoside in negative ion mode. $\mathrm{A}_{1}: \mathrm{GRg}_{1}(\mathrm{Rt} 2.40, m / z 845.4856), \mathrm{B}_{1}: \mathrm{GRe}(\mathrm{Rt} 2.36, m / z$ 991.5491), $\mathrm{C}_{1}$ : GRd (Rt 14.72, $\mathrm{m} / z$ 991.5526), $\mathrm{D}_{1}$ : GRo (Rt 9.34, $\mathrm{m} / z$ 955.4781), $\mathrm{E}_{1}$ : GRc (Rt 10.09, $\mathrm{m} / z$ 1077.5908), $\mathrm{F}_{1}: \mathrm{GRb}_{2}(\mathrm{Rt} 11.49, m / z 1077.5840), \mathrm{G}_{1}$ : $\mathrm{GRb}_{3}$ (Rt 11.97, $m / z$ 1077.5867), $\mathrm{H}_{1}: \mathrm{GRb}_{1}$ (Rt 8.78, $m / z$ 1107.6164), $\mathrm{M}_{1}$ : MalonylGRc (Rt 10.56, $m / z$ 1163.5669), $\mathrm{N}_{1}:$ MalonylGRb $_{1}(\mathrm{Rt} 12.35, m / z$ 1163.5894), $\mathrm{X}_{1}:$ MalonylGRb $_{2}$ (Rt 9.53, $\mathrm{m} / z$ 1193.5908), $\mathrm{Y}_{1}: \mathrm{GRa}_{2}$ (Rt 8.34, $\mathrm{m} / z$ 1209.6553), $\mathrm{Z}_{1}: \mathrm{GRa}_{1}(\mathrm{Rt} 8.37, \mathrm{~m} / z$ 1209.6318).

\section{Abbreviations}

AUC:

B-Group:

BCRP:

C-Group:

CYP450:

DDAs:

F-Group:

MRT:

MDAs:

M-Group:

MRP2:

P-gp:

PLS-DA:

PCA:
Area under the Curve

Blank group

Breast cancer resistance protein

Codecoction group

Cytochrome P450

Diester diterpenoid alkaloids

Fuzi decoction group

Mean Residence Time

Monoester diterpenoid alkaloids

Mixed decoction group

Multidrug resistance-associated protein isoform 2

P-glycoprotein

Partial least-squares-discriminant analysis

Principal component analysis
R-Group: Renshen decoction group

SFD: $\quad$ Shenfu Decoction

TCM: $\quad$ Traditional Chinese Medicine

UPLC/Q-TOF-MS: Ultra Performance Liquid

Chromatography/Quadrupole

Time-of-Flight Mass Spectrometry.

\section{Conflict of Interests}

The authors declare no conflict of interests.

\section{Authors' Contribution}

Jia-le He and Jia-wei Zhao contributed equally to this work. Yu-guang Wang, Qian-de Liang, Hong-ling Tan, Cheng-rong Xiao, and Xiang-lin Tang carried out the animal surgery and 

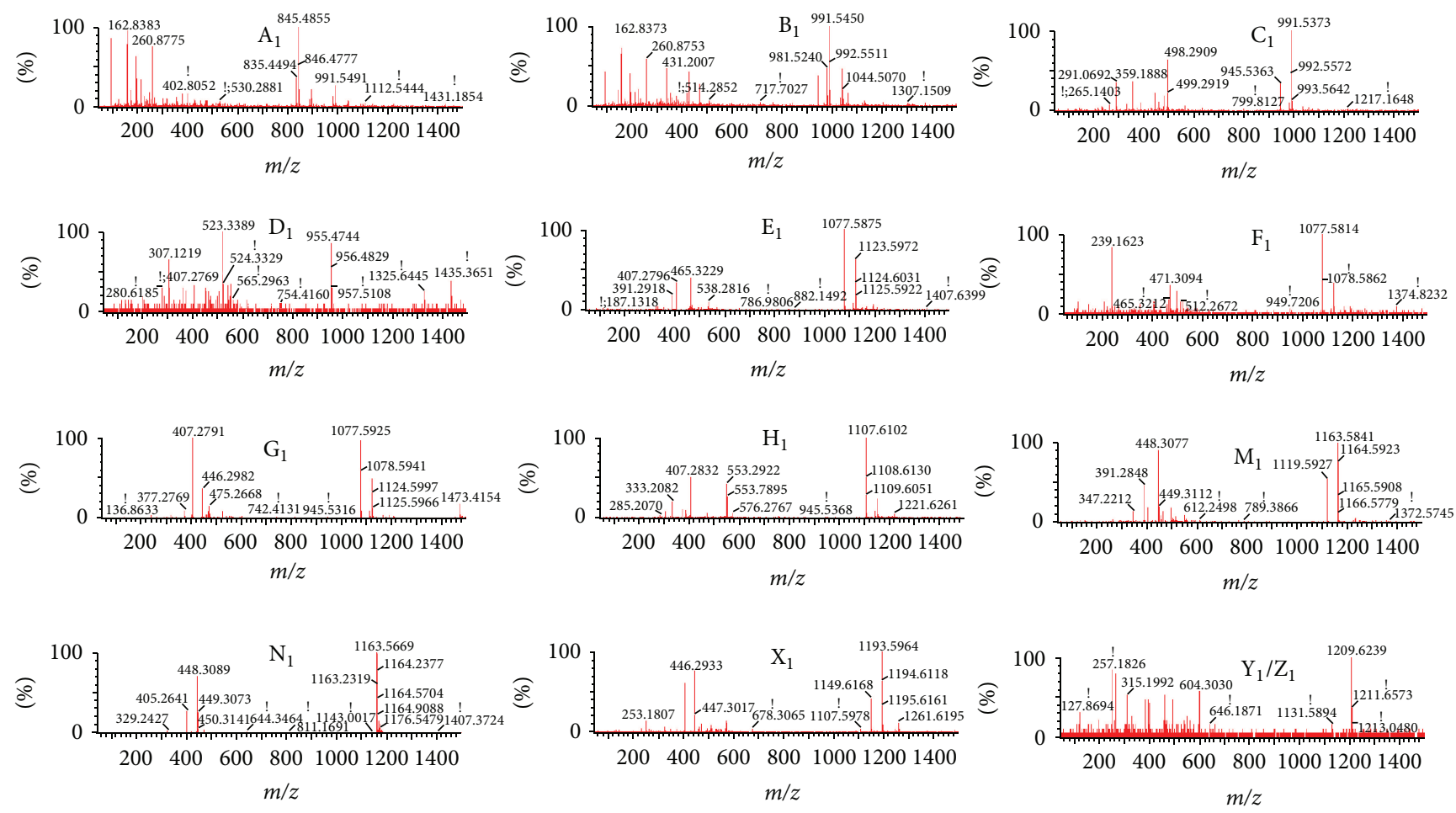

FIGURE 10: Mass spectrogram of ginsenoside in negative ion mode. $\mathrm{A}_{1}$ : GRg $\left(\right.$ Rt 2.40, $\mathrm{m} / z$ 845.4856), $\mathrm{B}_{1}$ : GRe (Rt 2.36, $m / z$ 991.5491), $\mathrm{C}_{1}$ : GRd (Rt 14.72, $m / z$ 991.5526), $\mathrm{D}_{1}$ : GRo (Rt 9.34, $\mathrm{m} / z$ 955.4781), $\mathrm{E}_{1}$ : GRc (Rt 10.09, $\mathrm{m} / z$ 1077.5908), $\mathrm{F}_{1}$ : GRb ${ }_{2}(\mathrm{Rt} 11.49, m / z 1077.5840), \mathrm{G}_{1}$ : $\mathrm{GRb}_{3}$ (Rt 11.97, $m / z$ 1077.5867), $\mathrm{H}_{1}: \mathrm{GRb}_{1}$ (Rt 8.78, $m / z$ 1107.6164), $\mathrm{M}_{1}:$ MalonylGRc (Rt 10.56, $m / z$ 1163.5669), $\mathrm{N}_{1}:$ MalonylGRb $_{1}\left(\mathrm{Rt}_{12.35}, \mathrm{~m} / z\right.$ 1163.5894), $\mathrm{X}_{1}:$ MalonylGRb $_{2}$ (Rt 9.53, $\mathrm{m} / z$ 1193.5908), $\mathrm{Y}_{1}: \mathrm{GRa}_{2}$ (Rt 8.34, $\mathrm{m} / z$ 1209.6553), $\mathrm{Z}_{1}: \mathrm{GRa}_{1}(\mathrm{Rt} 8.37, \mathrm{~m} / z$ 1209.6318).

the blood collection. Zeng-chun Ma and Yue Gao contributed to critical review of the paper.

\section{Acknowledgments}

This work was supported by the National Natural Science Foundation of China (no. 81274127) and the National Basic Research Program of China (“973" Project nos. 2012CB518402 and 2011CB505304).

\section{References}

[1] J.-H. Chen, C.-Y. Lee, B.-C. Liau, M.-R. Lee, T.-T. Jong, and S.-T. Chiang, "Determination of aconitine-type alkaloids as markers in fuzi (Aconitum carmichaeli) by LC/(+)ESI/MS 3 ," Journal of Pharmaceutical and Biomedical Analysis, vol. 48, no. 4, pp. 11051111, 2008.

[2] Z.-J. Guo and C.-S. Li, "Therapeutic effects of shenfu injection on post-cardiac arrest syndrome," Chinese Journal of Integrative Medicine, vol. 19, no. 9, pp. 716-720, 2013.

[3] J. Luo, S. Min, K. Wei, and J. Cao, "Ion channel mechanism and ingredient bases of Shenfu Decoction's cardiac electrophysiological effects," Journal of Ethnopharmacology, vol. 117, no. 3, pp. 439-445, 2008.

[4] X. J. Wang, "Studies on serum pharmacochemistry of traditional Chinese medicine," World Science and Technology, vol. 4, no. 2, pp. 1-5, 2002.
[5] S.-S. Zhou, Z.-C. Ma, Y. Gao, and et al, "UPLC-TOF/MS based chemical profiling approach to evaluate toxicity-attenuated chemical composition in combination of ginseng and Radix Aconiti Praeparata," Acta Pharmaceutica Sinica, vol. 46, no. 12, pp. 1488-1492, 2011.

[6] H. Li, Y.-G. Wang, Z.-C. Ma et al., "Effect of Shenfu injection on CYP450s of rat liver," Acta Pharmaceutica Sinica, vol. 48, no. 5, pp. 728-733, 2013.

[7] Y.-G. Wang, H.-S. Liu, Y. Gao et al., "Screening of pregnane $\mathrm{X}$ receptor activation from ginsenosides," Acta Pharmaceutica Sinica, vol. 48, no. 1, pp. 144-148, 2013.

[8] S. S. Zhou, Z. C. Ma, Q. Liang et al., "UPLC/Q-TOF-MS based chemical profiling approach to evaluate chemical composition of augmentation toxicity in combination of radix aconiti and pinellia praeparata," Acta Chimica Sinica, vol. 70, no. 3, pp. 284290, 2012.

[9] S. S. Zhou, Z. C. Ma, Y. Gao et al., "UPLC/Q-TOF-MS based chemical profiling approach to evaluate ginsenoside composition in combination of ginseng and radix ophiopogonis," Journal of Chinese Mass Spectrometry Society, vol. 34, no. 2, pp. 88-91, 2013.

[10] J.-L. He, S.-S. Zhou, Z.-C. Ma et al., "To evaluate the meterial basis of Shenfu injection based on UPLC-Q-TOF/MS," Chinese Pharmacological Bulletin, vol. 30, no. 3, pp. 429-433, 2014.

[11] L. Ye, X. S. Yang, Z. Q. Yang et al., "The role of efflux transporters on the transport of highly toxic aconitine, mesaconitine, hypaconitine, and their hydrolysates, as determined in cultured Caco-2 and transfected MDCKII cells," Toxicology Letters, vol. 216, no. 2-3, pp. 86-99, 2013. 
[12] N. Li, R. Tsao, Z. G. Sui, J. Ma, Z. Lie, and Z. Lie, "Intestinal transport of pure diester-type alkaloids from an aconite extract across the Caco-2 cell monolayer model," Planta Medica, vol. 78, no. 7, pp. 692-697, 2012.

[13] C. Yang, Z. Li, T. Zhang, F. Liu, J. Ruan, and Z. Zhang, "Transcellular transport of aconitine across human intestinal Caco-2 cells," Food and Chemical Toxicology, vol. 57, pp. 195200, 2013.

[14] A. Ameri, "The effects of Aconitum alkaloids on the central nervous system," Progress in Neurobiology, vol. 56, no. 2, pp. 211235, 1998.

[15] A. M. Bello-Ramírez, J. Buendía-Orozco, and A. A. NavaOcampo, "A QSAR analysis to explain the analgesic properties of Aconitum alkaloids," Fundamental and Clinical Pharmacology, vol. 17, no. 5, pp. 575-580, 2003.

[16] P. J. Tong, C. L. Wu, X. F. Wang et al., "Development and assessment of a complete-detoxication strategy for Fuzi (lateral root of Aconitum carmichaeli) and its application in rheumatoid arthritis therapy," Journal of Ethnopharmacology, vol. 146, no. 2, pp. 562-571, 2013.

[17] N. Guo, M. T. Liu, D. W. Yang et al., "Quantitative LC-MS/MS analysis of seven ginsenosides and three aconitum alkaloids in Shen-Fu decoction," Chemistry Central Journal, vol. 7, no. 1, article 165, 7 pages, 2013.

[18] L. Tang, Y. Gong, C. Lv, L. Ye, L. Liu, and Z. Liu, "Pharmacokinetics of aconitine as the targeted marker of fuzi (Aconitum carmichaeli) following single and multiple oral administrations of fuzi extracts in rat by UPLC/MS/MS," Journal of Ethnopharmacology, vol. 141, no. 2, pp. 736-741, 2012.

[19] J.-M. Zhang, W. Liao, Y.-X. He, Y. He, D. Yan, and C.M. Fu, "Study on intestinal absorption and pharmacokinetic characterization of diester diterpenoid alkaloids in precipitation derived from Fuzi-Gancao herb-pair decoction for its potential interaction mechanism investigation," Journal of Ethnopharmacology, vol. 147, no. 1, pp. 128-135, 2013.

[20] Z. Li, R. Zhang, X. Wang, X. Hu, Y. Chen, and Q. Liu, "Simultaneous determination of seven ginsenosides in rat plasma by high-performance liquid chromatography coupled to time-offlight mass spectrometry: application to pharmacokinetics of Shenfu injection," Biomedical Chromatography, vol. 29, no. 2, pp. 167-175, 2015.

[21] H. Yang, L. Liu, W. Gao, K. Liu, L.-W. Qi, and P. Li, "Direct and comprehensive analysis of ginsenosides and diterpene alkaloids in Shenfu injection by combinatory liquid chromatographymass spectrometric techniques," Journal of Pharmaceutical and Biomedical Analysis, vol. 92, pp. 13-21, 2014.

[22] T. Tazawa, H.-Q. Zhao, Y. Li et al., "A new enzyme immunoassay for aconitine and its application to quantitative determination of aconitine levels in plasma," Biological and Pharmaceutical Bulletin, vol. 26, no. 9, pp. 1289-1294, 2003.

[23] F. Zhang, M.-H. Tang, L.-J. Chen et al., "Simultaneous quantitation of aconitine, mesaconitine, hypaconitine, benzoylaconine, benzoylmesaconine and benzoylhypaconine in human plasma by liquid chromatography-tandem mass spectrometry and pharmacokinetics evaluation of 'SHEN-FU' injectable powder,' Journal of Chromatography B: Analytical Technologies in the Biomedical and Life Sciences, vol. 873, no. 2, pp. 173-179, 2008.

[24] C.-Z. Wang, K. E. Kim, G.-J. Du et al., "Ultra-performance liquid chromatography and time-of-flight mass spectrometry analysis of ginsenoside metabolites in human plasma," The American Journal of Chinese Medicine, vol. 39, no. 6, pp. 1161$1171,2011$.
[25] C. X. Liu, Y. Z. Hou, X. L. Wang et al., "Clinical assessment of Shenfu injection loading in the treatment of patients with exacerbation of chronic heart failure due to coronary heart disease: study protocol for a randomized controlled trial," Trials, vol. 16, no. 1, article 222, 2015.

[26] Y. L. Zhao, J. B. Wang, X. J. Sun et al., "Microcalorimetry coupled with chemometric techniques for toxicity evaluation of Radix Aconiti Lateralis Preparata (Fuzi) and its processed products on Escherichia coli," Applied Microbiology and Biotechnology, vol. 98, no. 1, pp. 437-444, 2014.

[27] L. Ye, X.-S. Yang, L.-L. Lu et al., "Monoester-diterpene aconitum alkaloid metabolism in human liver microsomes: predominant role of CYP3A4 and CYP3A5," Evidence-based Complementary and Alternative Medicine, vol. 2013, Article ID 941093, 24 pages, 2013.

[28] Q. You, Z. C. Ma, Y. G. Wang et al., "Absorption characteristics of panax ginseng water extract in Caco-2 cell monolayer," Chinese Pharmacology Bulletin, vol. 29, no. 12, pp. 1711-1716, 2013.

[29] K. Wada, M. Nihira, H. Hayakawa, Y. Tomita, M. Hayashida, and Y. Ohno, "Effects of long-term administrations of aconitine on electrocardiogram and tissue concentrations of aconitine and its metabolites in mice," Forensic Science International, vol. 148, no. 1, pp. 21-29, 2005.

[30] W.-W. Peng, W. Li, J.-S. Li et al., "The effects of Rhizoma Zingiberis on pharmacokinetics of six Aconitum alkaloids in herb couple of Radix Aconiti Lateralis-Rhizoma Zingiberis," Journal of Ethnopharmacology, vol. 148, no. 2, pp. 579-586, 2013.

[31] J. Sun, W. Wu, Y. Y. Guo, Q. Qin, and S. Liu, "Pharmacokinetic study of ginsenoside Rc and simultaneous determination of its metabolites in rats using RRLC-Q-TOF-MS," Journal of Pharmaceutical and Biomedical Analysis, vol. 88, pp. 16-21, 2014.

[32] F. Zhao, L. Xu, and L. Xu, "Trains of thoughts and methods in studying substantial basis of effects of Chinese medicinal compounds," Chinese Journal of Integrated Medicine, vol. 27, no. 1, pp. 80-82, 2007. 


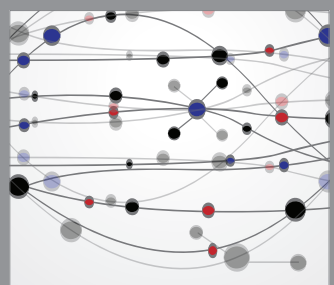

The Scientific World Journal
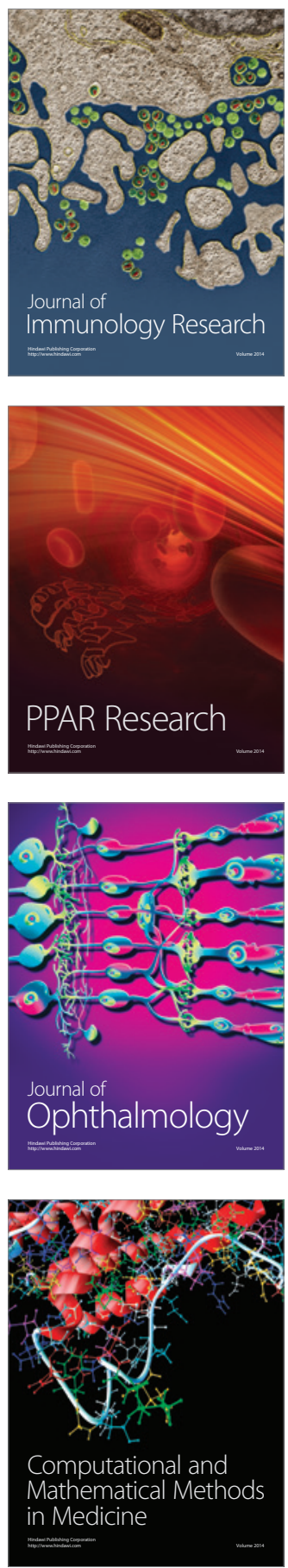

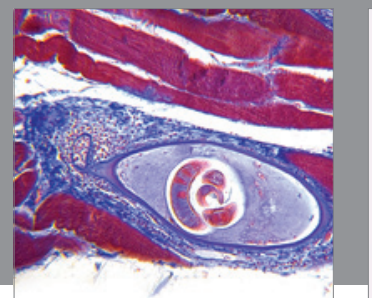

Gastroenterology

Research and Practice
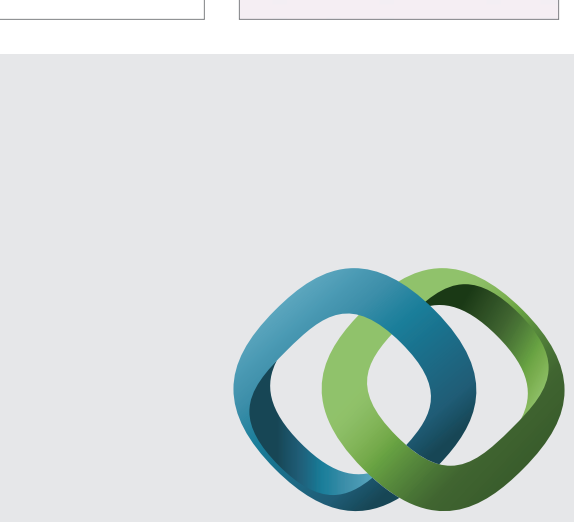

\section{Hindawi}

Submit your manuscripts at

http://www.hindawi.com
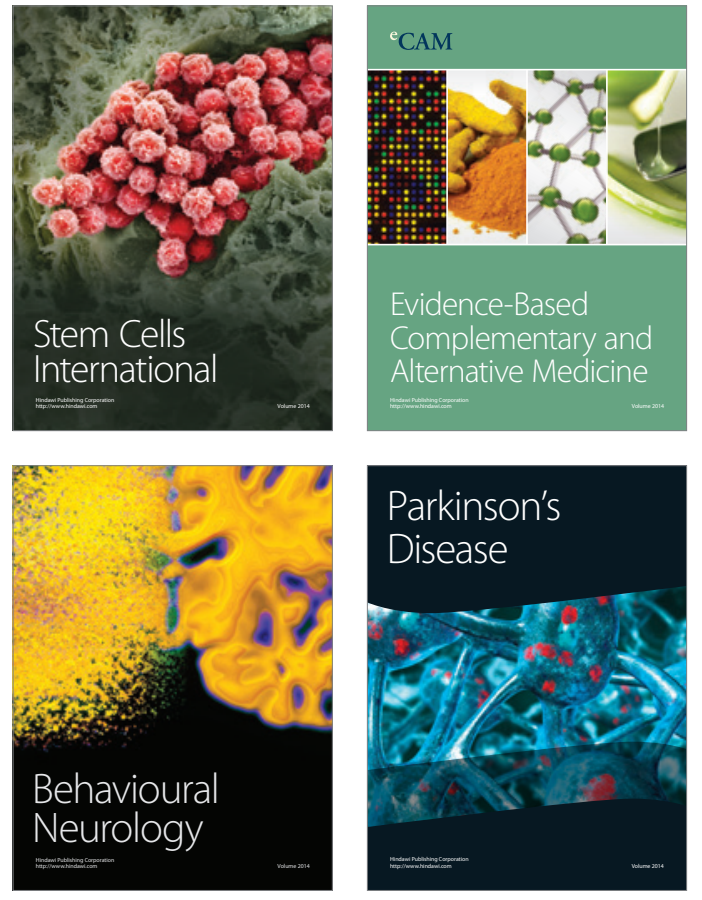
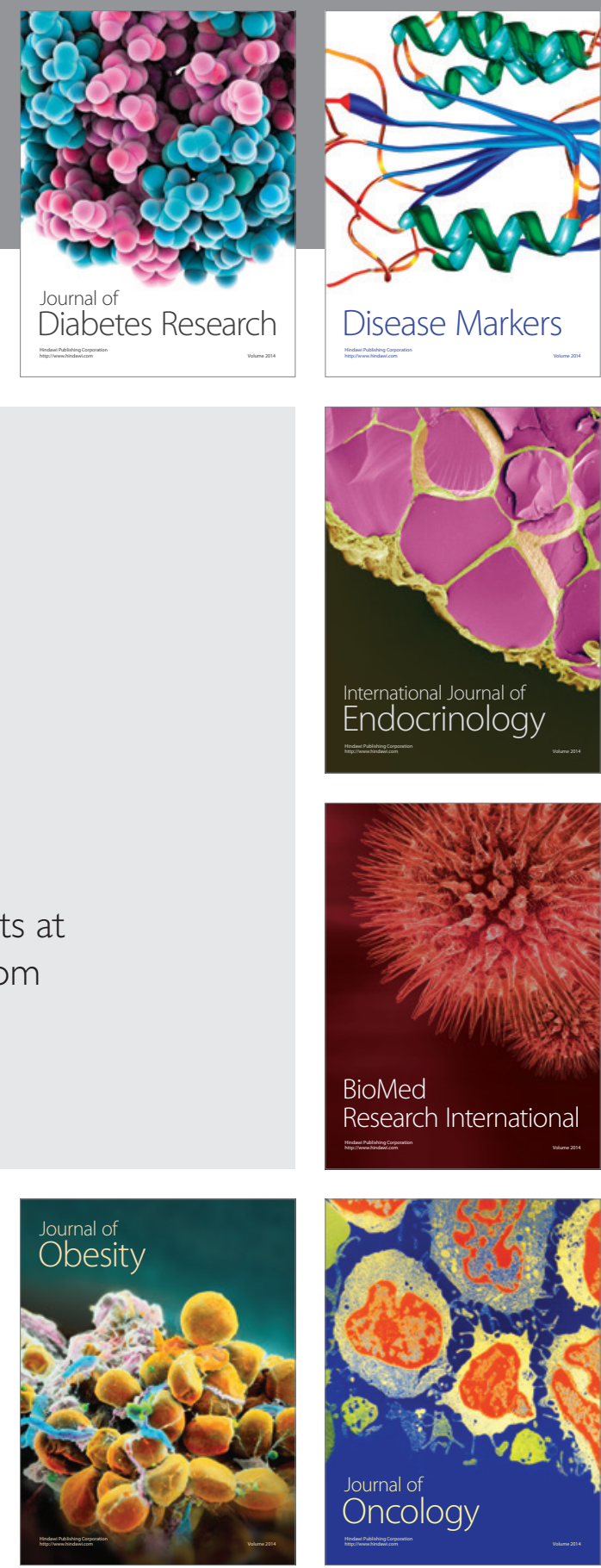

Disease Markers
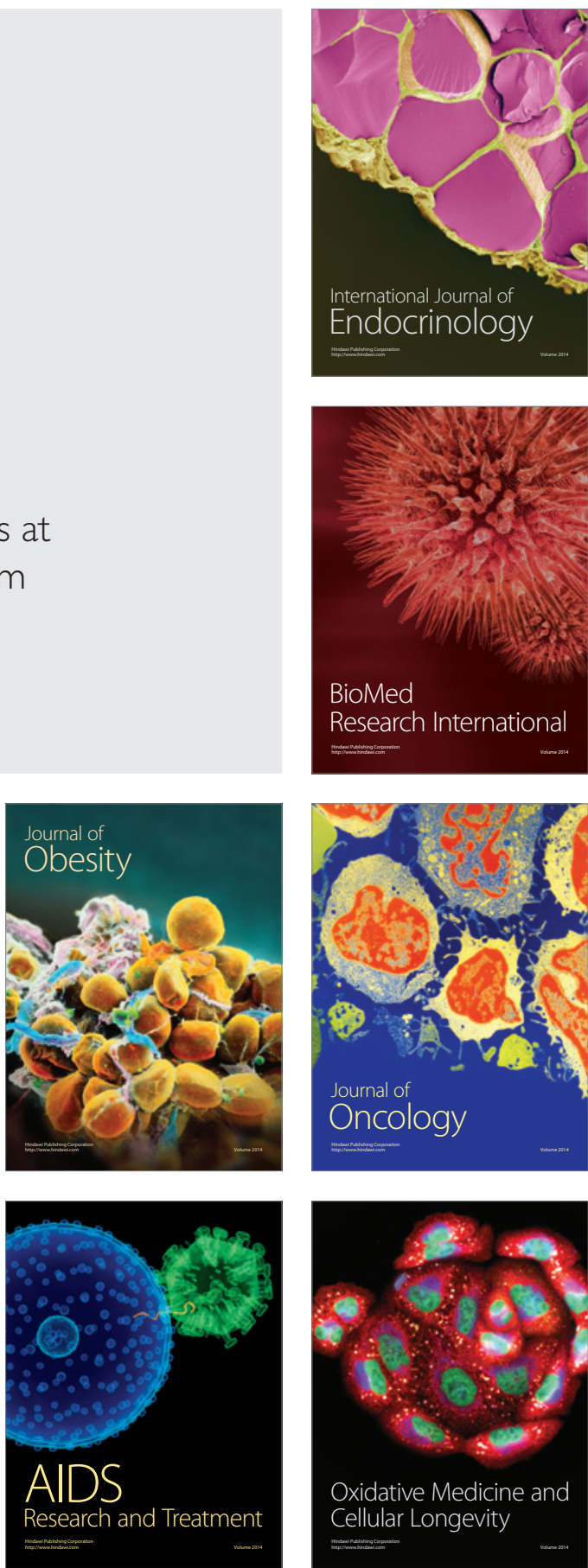